\title{
Propuesta de una asignatura de Diseño de Servidores para la especialidad de Tecnologías de Información
}

\author{
A. J. Rivera ${ }^{1}$, M. Espinilla ${ }^{1}$, A. Fernández ${ }^{1}$, J. Santamaría ${ }^{1}$, F. Charte ${ }^{2}$ \\ ${ }^{1}$ Departamento de Informática. Universidad de Jaén \\ \{arivera, mestevez, ahilario, jslopez\}@ujaen.es \\ ${ }^{2}$ Dpto. De Ciencias de Computación e Inteligencia Artificial. Universidad de Granada \\ francisco@fcharte.com
}

\begin{abstract}
Resumen. En este trabajo se presenta la asignatura de Diseño e Implantación de Servidores de la especialidad de Tecnologías de Información perteneciente a los estudios de Ingeniería en Informática de la Universidad de Jaén. El objetivo de esta asignatura es cubrir la formación que se establece en diferentes competencias relacionadas con en el desarrollo, despliegue e implantación de infraestructuras hardware necesarias para el soporte y funcionamiento de los sistemas de información actuales. Estos sistemas de información se caracterizan por presentar necesidades como acceso ubicuo, gran capacidad de cómputo o alta disponibilidad, entre otras. De esta manera la asignatura presenta un programa teórico/práctico donde se recorren desde principios básicos en el diseño de sistemas, su monitorización, referenciación o evaluación hasta conceptos de diseño actuales como alta disponibilidad, escalabilidad o balanceo de carga.
\end{abstract}

Palabras Clave: Diseño de servidores, monitorización, referenciación, evaluación, disponibilidad, escalabilidad, balanceo de carga.

\begin{abstract}
This paper presents the subject Design and Deployment of Servers belonging to the Information Technologies of the Computer Science Engineering at the University of Jaén. The objective of this subject is to provide the training established in the different competences related to the development and deployment of hardware infrastructures that supports the currently information systems. These information systems have characteristics such as ubiquitous access, high computational costs or high availability, among others. Thus, the subject addresses concepts from design of systems, monitoring, benchmarking or evaluation, to high availability, scalability or load balancing.
\end{abstract}

Keywords: Design of servers, monitoring, benchmarking, evaluation, high availability, scalability, load balancing. 


\section{Introducción}

En los últimos estudios realizados por Gartner (http://www.gartner.com) o Forrester (http://www.forrester.com) sobre nuevas tendencias en el campo de las Tecnologías de la Información encontramos que estas se corresponden con tecnologías como la computación en la nube, la inteligencia de negocios, la computación de grandes cantidades de información (Big Data), la computación ubicua, etc. Todas estas disciplinas tienen como factor común la necesidad de una infraestructura para la computación de altas prestaciones o supercomputación.

Entre las prioridades que se establecieron para el Espacio Europeo de Educación Superior (EEES) de la década actual en la Conferencia de Ministros europeos responsables de educación superior [1] podemos encontrar la empleabilidad. En esta prioridad se enfatiza la orientación de las enseñanzas del mercado hacia la vida y el mercado laboral destino de los egresados.

En este sentido se han introducido diferentes competencias en las recomendaciones para el diseño de los grados en Ingeniería en Informática. Algunas de estas competencias se recogen y desarrollan en la asignatura de Diseño e Implantación de Servidores perteneciente a la especialidad de Tecnologías de la Información del grado en Ingeniería en Informática de la Universidad de Jaén.

El objetivo de este artículo es presentar dicha asignatura para lo cual en la sección 2 se expondrá la especialidad en la que se encuadra, temporalidad o créditos ECTS. En la sección 3 se describirá la asignatura en sí: sus competencias, contenidos, actividades formativas, programas teórico y práctico, y bibliografía.

\section{El marco de la asignatura}

Cómo se ha mencionado la asignatura de Diseño e Implantación de Servidores se enmarca dentro de la especialidad de Tecnologías de Información de la Ingeniería en Informática de la Universidad de Jaén. Esta ingeniería se diseña conforme a las directrices del Ministerio de Educación para la profesión de Ingeniero Técnico en Informática en la Resolución 12977 de la Secretaria General de Universidades publicada en el Boletín Oficial del Estado (BOE) de 4 de Agosto de 2009.

La especialidad de Tecnologías de Información consta de 48 créditos ECTS que el alumno debe cursar obligatoriamente. El módulo se compone de tres materias y 8 asignaturas, su correspondencia se muestra en las Tabla 1. Temporalmente se imparte entre tercer y cuarto curso. Esta planificación se muestra en la Tabla 2. 
Tabla 1. Relación de materias y asignaturas que componen el módulo de Tecnologías de Información

\begin{tabular}{|c|c|c|c|}
\hline $\begin{array}{l}\text { Denominación de la } \\
\text { materia }\end{array}$ & Asignatura & ECTS & $\begin{array}{l}\text { Carácter de la } \\
\text { materia }\end{array}$ \\
\hline \multirow{3}{*}{ TECNOLOGÍAS WEB } & SISTEMAS MULTIAGENTE & \multirow{3}{*}{18} & \multirow{3}{*}{ Obligatorio } \\
\hline & TECNOLOGÍAS BASADAS EN WEB & & \\
\hline & $\begin{array}{l}\text { TECNOLOGÍAS PARA LA GESTIÓN DE } \\
\text { LA INFORMACIÓN }\end{array}$ & & \\
\hline \multirow[b]{2}{*}{ MULTIMEDIA } & SISTEMAS MULTIMEDIA & \multirow[b]{2}{*}{12} & \multirow[b]{2}{*}{ Obligatorio } \\
\hline & $\begin{array}{l}\text { PROCESAMIENTO DE INFORMACIÓN } \\
\text { VISUAL }\end{array}$ & & \\
\hline \multirow{3}{*}{$\begin{array}{l}\text { INFRAESTRUCTURAS } \\
\text { TECNOLÓGICAS }\end{array}$} & $\begin{array}{l}\text { DISEÑO E IMPLANTACIÓN DE } \\
\text { SERVIDORES }\end{array}$ & \multirow{3}{*}{18} & \multirow{3}{*}{ Obligatorio } \\
\hline & $\begin{array}{l}\text { REDES E INFRAESTRUCTURAS DE } \\
\text { COMUNICACIONES }\end{array}$ & & \\
\hline & $\begin{array}{l}\text { DESARROLLO SOFTWARE PARA } \\
\text { DISPOSITIVOS MÓVILES }\end{array}$ & & \\
\hline
\end{tabular}

Tabla 2. Distribución temporal del módulo de Tecnologías de Información

\begin{tabular}{|l|l|l|}
\hline Asignatura & Curso & Semestre \\
\hline SISTEMAS MULTIMEDIA & 3 & 2 \\
\hline TECNOLOGÍAS BASADAS EN WEB & 3 & 2 \\
\hline DESARROLLO SOFTWARE PARA DISPOSITIVOS MÓVILES & 3 & 2 \\
\hline SISTEMAS MULTIAGENTE & 3 & 2 \\
\hline PROCESAMIENTO DE INFORMACIÓN VISUAL & 3 & 2 \\
\hline DISEÑO E IMPLANTACIÓN DE SERVIDORES & 4 & 1 \\
\hline REDES E INFRAESTRUCTURAS DE COMUNICACIONES & 4 & 1 \\
\hline TECNOLOGÍAS PARA LA GESTIÓN DE LA INFORMACIÓN & 4 & 1 \\
\hline OPTATIVA 1 & 4 & - \\
\hline OPTATIVA 2 & 4 & - \\
\hline
\end{tabular}

\section{Descripción de la asignatura}

Tal y como aparece en la ficha VERIFICA, los datos básicos de la asignatura Diseño e Implantación de Servidores se resumen en que es una asignatura de la especialidad de Tecnologías de la Información en la Ingeniería en Informática de la Universidad de Jaén. Dispone de 6 créditos ECTS, se imparte en el primer semestre de cuarto curso y tiene un carácter obligatorio. A continuación, en la sección 3.1 se describen las capacidades contenidos descriptivos y actividades formativas de la asignatura, en la sección 3.2 los programas teóricos y prácticos, en la sección 3.3 la metodología de evaluación y en la sección 3.4 las recomendaciones bibliográficas. 


\subsection{Capacidades, contenidos y actividades formativas}

Las capacidades de la especialidad que según la ficha VERIFICA el alumno deberá adquirir son:

- CTI2: Capacidad para seleccionar, diseñar, desplegar, integrar, evaluar, construir, gestionar, explotar y mantener las tecnologías de hardware, software y redes, dentro de los parámetros de coste y calidad adecuados.

- CTI5: Capacidad para seleccionar, desplegar, integrar y gestionar sistemas de información que satisfagan las necesidades de la organización, con los criterios de coste y calidad identificados.

Los contenidos descriptivos que aparecen en la ficha para la asignatura son:

- Instalación, configuración, administración y mantenimiento de servidores.

- Monitorización y Evaluación de prestaciones.

- Conexión remota y Virtualización.

- Hardware para servidores: placas base, procesadores, almacenamiento e interconexión.

- Centros de Proceso de Datos.

- Seguridad y normativas.

Más pormenorizadamente las actividades formativas, con su contenido en créditos ECTS, su metodología de enseñanza-aprendizaje, y su relación con las competencias que debe adquirir el estudiante se describen en la Tabla 3.

Tabla 3: Actividades formativas, con su contenido en créditos ECTS, su metodología de enseñanza-aprendizaje, y su relación con las competencias

\begin{tabular}{|l|l|l|l|l|}
\hline $\begin{array}{l}\text { Actividades y } \\
\text { Metodologías }\end{array}$ & $\begin{array}{l}\text { Créditos } \\
\text { ECTS }\end{array}$ & $\begin{array}{l}\text { Horas } \\
\text { presenciales }\end{array}$ & $\begin{array}{l}\text { Horas trabajo } \\
\text { autónomo del } \\
\text { alumno }\end{array}$ & $\begin{array}{l}\text { Competencia/s } \\
\text { (códigos) }\end{array}$ \\
\hline $\begin{array}{l}\text { Clases expositivas } \\
\text { en gran grupo: } \\
\text { M1.1, M1.2, } \\
\text { M1.4, M1.5 }\end{array}$ & 2,5 & 25 & 37,5 & CTI2, CTI5 \\
\hline $\begin{array}{l}\text { Clases en grupos } \\
\text { de prácticas: } \\
\text { M2.1, M2.2, } \\
\begin{array}{l}\text { M2.4, M2.5, } \\
\text { M2.6, M2.7 }\end{array}\end{array}$ & 3 & 30 & 45 & CTI2, CTI5 \\
\hline $\begin{array}{l}\text { Tutorías } \\
\text { colectivas e } \\
\text { individuales: } \\
\text { M3.1, M3.4, } \\
\text { M3.5, M3.6 }\end{array}$ & 0,5 & 5 & 7,5 & CTI2, CTI5 \\
\hline Totales & 6 & 60 & 90 & \\
\hline
\end{tabular}

Concretamente las posibles metodologías a utilizar se enumeran en la Tabla 4. 
Tabla 4: Posibles metodologías a utilizar

\begin{tabular}{|c|c|c|}
\hline Actividades & Metodología & Código \\
\hline \multirow{5}{*}{$\begin{array}{l}\text { Clases expositivas en } \\
\text { gran grupo }\end{array}$} & Clases magistrales & M1.1 \\
\hline & $\begin{array}{l}\text { Exposición de teoría y } \\
\text { ejemplos generales }\end{array}$ & M1.2 \\
\hline & Actividades introductorias & M1.3 \\
\hline & Conferencias, etc & M1.4 \\
\hline & Otros & M1.5 \\
\hline \multirow{8}{*}{$\begin{array}{l}\text { Clases en grupos de } \\
\text { prácticas }\end{array}$} & Actividades practicas & M2.1 \\
\hline & Seminarios & M2.2 \\
\hline & Debates & M2.3 \\
\hline & Laboratorios & M2.4 \\
\hline & Aulas de informática & M2.5 \\
\hline & Resolución de ejercicios & M2.6 \\
\hline & Presentaciones/exposiciones & M2.7 \\
\hline & Otros & M2.8 \\
\hline \multirow[t]{6}{*}{$\begin{array}{l}\text { Tutorías } \\
\text { colectivas/individuales }\end{array}$} & $\begin{array}{l}\text { Supervisión de trabajos } \\
\text { dirigidos }\end{array}$ & M3.1 \\
\hline & Seminarios & M3.2 \\
\hline & Debates & M3.3 \\
\hline & Aclaración de dudas & M3.4 \\
\hline & $\begin{array}{l}\text { Comentarios de trabajos } \\
\text { individuales }\end{array}$ & M3.5 \\
\hline & Presentaciones/exposiciones & M3.6 \\
\hline
\end{tabular}

\subsection{Programa teórico y práctico}

Tanto los contenidos descriptivos como las competencias establecidas para la asignatura en la ficha VERIFICA, citados en el apartado anterior, intentan delinear una asignatura que recoja la formación necesaria que debe de tener un alumno que curse la especialidad de Tecnologías de Información en el ámbito del diseño de las infraestructuras de computo actuales.

Esta formación quedará totalmente delimitada con los siguientes programas de teoría y prácticas. Concretamente el temario de teoría que se establece para la asignatura es:

Tema 1. Introducción

1.1. ¿Qué es un servidor?

1.2. Tipos de servidores

1.3. Caracterización de servidores

1.4. Rendimiento y evaluación

1.5. Comparación de prestaciones 
Tema 2: Internet. Administración y servicios

2.1. Introducción

2.2. Direccionamiento IP

2.3. Enrutamiento

2.4. DHCP

2.5. DNS

2.6. NAT

Tema 3: Monitorización y referenciación

3.1. Introducción

3.2. Tipos de monitores y sus parámetros

3.3. Herramientas de monitorización

3.4. Índices de referenciación

3.5. Software de referenciación

Tema 4: Servidores de altas prestaciones

4.1. Introducción

4.2. Alta disponibilidad

4.3. Escalabilidad

4.4. Balanceo de carga

4.5. Virtualización

Tema 5: Análisis operacional de servidores

5.1. Introducción

5.2. Redes de colas

5.3. Variables y leyes operacionales

5.4. Límites asintóticos del rendimiento

5.5. Algoritmos de resolución de modelos de redes de colas

Tema 6: Hardware de servidores y Centros de Proceso de Datos

6.1. Introducción

6.2. Hardware de servidores

6.3. Sistemas de almacenamiento

6.4. Formatos de servidores

6.5. Centros de proceso de Datos

El programa de prácticas diseñado intentará apuntalar los conceptos explicados en teoría y tendrá como hándicap una sincronización adecuada con esta. Concretamente se propone el siguiente programa de prácticas:

Práctica 1: Introducción a la administración de servidores (2 sesiones)

1.1. Herramientas para la administración servidores

1.2. Configuración básica de redes

Práctica 2: Configuración de redes y routers (1 sesión)

2.1. Configuración de redes

2.2. Tablas de enrutamiento 
Práctica 3: Configuración de DHCP y DNS (1 sesión)

3.1 Configuración de un servidor dnsmasq

Práctica 4: Configuración de IPTables (1 sesión)

4.1. Cortafuegos

4.2. Enmascaramiento

4.3. Redireccionamiento

Práctica 5: Virtualización (3 sesiones)

5.1. Introducción a la virtualización

5.2. Virtualización de servidores

Práctica 6: Monitorización del sistema (1 sesión)

6.1. Herramientas básicas de monitorización del sistema

6.2. Monitorización con SAR

Práctica 7: Referenciación (1 sesión)

6.1. Protocolos y plataformas de referenciación

Práctica 8: Proyecto de prácticas (5 sesiones)

Las 7 primeras prácticas tienen un formato de desarrollo clásico donde hay un material teórico que es preparado y explicado por el profesor. Tras esto los alumnos pasan al desarrollo práctico conducidos por el correspondiente guion.

La última práctica se plantea como proyecto de la asignatura y consistirá en la instalación, configuración y puesta en marcha de un servidor de altas prestaciones. Concretamente al alumno se le propones varias tecnologías que caracterizan los servidores de altas prestaciones actuales como: alta disponibilidad, balanceo de carga, cluster de computación, ... y se le pide que desarrolle e implante una. El formato de las sesiones cambia completamente, ya que ahora la idea es que el alumno no esté tan dirigido y que busque la documentación necesaria para llevar a cabo el proyecto.

Todas las prácticas, incluyendo el proyecto, se desarrollan sobre Linux, concretamente utilizando la distribución CentOS, la cual dispone de los paquetes software adecuados para el desarrollo de un servidor de altas prestaciones.

\subsection{Metodología de evaluación}

La metodología de evaluación es uno de los componentes más importantes que definen una asignatura ya que van a caracterizar fuertemente el proceso de enseñanzaaprendizaje de un alumno.

Así, el diseño de sistemas de evaluación adecuados va a incentivar el estudio habitual en el alumno y le va a servir para autoevaluarse en la compresión de los 
contenidos teóricos o prácticos correspondientes. Para el profesor también van a ser un instrumento muy valioso ya que le permiten conocer el nivel de comprensión de los alumnos.

En este sentido, potenciando el trabajo individual y diario del alumno, se ha diseñado la metodología de evaluación de la asignatura, la cual se muestra en la Tabla 5 .

Tabla 5. Metodología de evaluación

\begin{tabular}{|l|l|l|l|}
\hline Aspecto & Criterios & Instrumento & Peso \\
\hline $\begin{array}{l}\text { Asistencia y } \\
\text { Participación }\end{array}$ & $\begin{array}{l}\text {-Participación activa en } \\
\text { la clase. } \\
\text {-Participación en el } \\
\text { trabajo grupal }\end{array}$ & $\begin{array}{l}\text { Observación y notas } \\
\text { del profesor }\end{array}$ & $10 \%$ \\
\hline $\begin{array}{l}\text { Conceptos teóricos } \\
\text { de la materia }\end{array}$ & $\begin{array}{l}\text {-Dominio de los } \\
\text { conocimientos teóricos } \\
\text { yoperativos de la } \\
\text { materia. }\end{array}$ & $\begin{array}{l}\text { Examen teórico } \\
\text { (prueba objetiva de } \\
\text { respuesta breve y } \\
\text { prueba objetiva tipo } \\
\text { test) }\end{array}$ & $35 \%$ \\
\hline $\begin{array}{l}\text { Conceptos prácticos } \\
\text { de la materia }\end{array}$ & $\begin{array}{l}\text {-Dominio de los } \\
\text { conocimientos } \\
\text { prácticos de la } \\
\text { materia }\end{array}$ & $\begin{array}{l}\text { Entrega de guiones de } \\
\text { sesiones prácticas. } \\
\text { Pruebas de tipo test } \\
\text { periódicas }\end{array}$ & $40 \%$ \\
\hline $\begin{array}{l}\text { Realización de } \\
\text { trabajos o casos }\end{array}$ & $\begin{array}{l}\text {-Entrega y exposición } \\
\text { de trabajos } \\
\text { investigación. En cada } \\
\text { trabajo se analizará: } \\
\text {-Estructura y } \\
\text { calidad de la } \\
\text { documentación } \\
\text {-Originalidad }\end{array}$ & Trabajo por grupos & $15 \%$ \\
\hline
\end{tabular}

\subsection{Bibliografía}

La bibliografía de una asignatura del ámbito de tecnologías de información cómo es la actual suele pivotar sobre una serie de recursos más o menos clásicos donde se recogen conceptos fundamentales relacionados y sobre otra serie de recursos más actuales, más técnicos y normalmente disponibles en la web.

Este es el caso de la asignatura de Diseño e Implantación de Servidores, donde se propone para el programa teórico una bibliografía compuesta de libros clásicos de diseño de sistemas o de evaluación y modelado de éstos [3,5,7]. También y para cubrir las últimas tendencias en estas materias se proponen obras cómo $[2,4]$

Para el programa de prácticas además de un best-seller en administración de sistemas en Linux como es [6] se propone la documentación electrónica disponible en el sitio web de Red Hat para la administración de sistemas. https://access.redhat.com/site/documentation/en-US/Red_Hat_Enterprise_Linux/ 


\section{Referencias}

1. Comunicado de la Conferencia de Ministros europeos responsables de educación superior, Lovaina / Louvain-la-Neuve, 28-29 de abril de 2009. http://viceees.ujaen.es/files_viceees/Comunicado-Lovaina-2009.pdf

2. Alger, D.: Build the best data center facility for your business. Cisco Press, (2012)

3. Jain, R.: The art of computer systems performance analysis: techniques for experimental design, measurement. John Wiley, (1991)

4. Josyula, V., Orr. M., Page, G.: Cloud computing : automating the virtualized data center. Cisco Pres, (2011)

5. Lilja, D.: Measuring computer performance: a practitioner's guide. Cambridge University Press, (2000)

6. Nemeth, E., Snyder, G., Hein, T., Whaley, B.: Unix and Linux system administration handbook. 4th ed. Upper Saddle River, Prentice Hall, (2011)

7. Molero, X.: Evaluación y modelado del rendimiento de los sistemas informáticos. Prentice Hall, (2004) 
Enseñanza y Aprendizaje de Ingeniería de Computadores. Número 4, 2014 